\title{
PROYECCIONES
}

Revista de Matemática Vol. 10 Pág. 27-34

1791 - ISSN 0716-0917

Jniversidad Católica nol Norte

Mitolagosta - Chile

\section{ON THE COERCIVENESS PROPERTY OF THE BIHARMONIC OPERATOR}

\author{
Gabriel N. Gatica
}

We consirle" the weak formation of the himmmin exuation under two different kinks of homikin won ditions. It is shown, in one case, that the coercivenesc of the bitinear form associater inm be easity deduced by ueing the continuow- deprndence result for the laplace equation with Dirivh. let data. In the second case, a genemalised loincare inequatity is readily entoyed.

\section{INTRODUCTION}

Let $\Omega$ be a bounded domain in the plane with smooth boundary For any integer $m, H^{m}(\Omega)$ and $H_{0}(\Omega)$ denote the usual Sobolev spaces of order iII $[4],[5]$. Similarly, for $s \in R, H^{S}\left(x_{n}\right)$ denotes the corresponding sobolev space on the boundary, $[5]$. Now, given $f \in L^{2}(2), g \in H^{3 / 2}\left(x_{2}\right)$, $h_{1} 6 H^{-1 / 2}\left(x_{2}\right)$ and $h_{2} \in H^{-3 / 2}\left(x_{2}\right)$, we define the following BVPs

$$
\begin{aligned}
\Delta^{2} u & =f, \text { in } \Omega \\
u & =g, \text { on } a \\
M[u] & =h_{1} \text {, on } a
\end{aligned}
$$

and

$$
\begin{aligned}
& \Delta^{2} u=f, \text { in } \Omega \\
& M[u]=h_{1}, \text { on } \gamma \text { ? } \\
& M[u]=h_{2}, \text { on } x ?
\end{aligned}
$$


where $\mathrm{M}$ and $\mathrm{N}$ are the boundary operators given by

$$
M[u]:=i \Delta u+(1-\sigma) \frac{\partial^{2} u}{\partial n^{2}}
$$

and

$N[u]:=-\frac{\partial \Delta u}{\partial n}+(1-\sigma) \frac{\partial}{\partial T}\left[\frac{\partial^{2} u}{\partial x^{2}} n_{x} n_{y}-\frac{\partial^{2} u}{\partial x \partial y}\left(n_{x}^{2}-n_{y}^{2}\right)-\frac{\partial^{2} u}{\partial y^{2}} n_{x} n_{y}\right]$

Here, $n:=\left(n_{x}, n_{y}\right)$ is the outward normal to $x_{2}, x:=(x, y)$ is a generic vector in $R^{2}, \frac{\partial}{\partial T}$ denotes differentiation with respect to the arc length, and o is the ${ }^{\partial T}$ poisson coefficient which is assumed to be $0 \leqslant 0<1$.

$[2],[6]$.

The following version of the Lax-Milgram lemma will be utilized

Lemma 1 .

Let $B$ be a bilinear form in a real Hilbert $\|$ with norm $\|\cdot\|_{H}$. Suppose that there exist positive constants $\alpha$, $\beta$ such that

$$
|B(v, w)| \leqslant \alpha\|v\|_{H}\|w\|_{H}
$$

for allv, wEH, and

$$
R\|v\|_{H}^{2} \leqslant B(v, v)
$$

for all $\vee \in H$.

Then, for every bounded linear functional $F \in \mathbb{H}^{\prime}$, there exists $A$ unique $u G H$ such that

$$
B(u, v)=F(v)
$$

for all $\vee \in H$.

Moreover, the following estimate holds

$$
\|u\|_{H} \leqslant \frac{1}{p}\|F\|_{H^{\prime}}
$$

Proof:

\section{See [2]: Theoren 1.1.3.}

The inequality (4) is called the coerciveness property or H ellipticity of $B$. Also, (5) is commonly refered as the continuous-dependence result. It is worth remarking that a more general lemula than the above one can be seen in ([4]: Theoren 14.1) and also in ([1]: Theorem $5.2 .1)$. 


\section{THE WEAK FORMUATIONS}

First, we turn our interest to the BVI (1) which physically represents the deflection of a horizontal vertically loaded plate with prescribed deformation and moments along the brumbry.

By writing

$$
\begin{gathered}
\Lambda^{2} u=\frac{\partial^{2}}{\partial x^{2}}\left[\frac{\partial^{2} u}{\partial x^{2}}\right]+2(1-\sigma) \frac{\partial^{2}}{\partial x \partial v}\left[\frac{\partial^{2} u}{\partial x \cdot y}\right]+\sigma \frac{\partial^{2}}{\partial x^{2}} \cdot\left[\frac{\partial^{2} u}{\partial y^{2}}\right]+ \\
\sigma \frac{\partial^{2}}{\partial y^{2}}\left[\frac{\partial^{2} u}{\partial x^{2}}\right]+\frac{\partial^{2}}{\partial y^{2}}\left[\frac{\partial^{2} u}{\partial y^{2}}\right]
\end{gathered}
$$

and then, applying integration by parts we obtain ifter a few computations (see [6]: chapter 23)

$$
\int v \wedge^{2} u d x=B(u, v)-\int_{x_{2}} v N[u] d T-\int_{2} \frac{d v}{\partial n} M[u] d T
$$

for all u $\in H^{4}(\Omega), v \in H^{2}(\Omega)$, where $B$ is the bilinear form given by

$B(u, v):=\int_{u}\left\{\right.$ (rAuAv $\left.+(1-\sigma)\left[\frac{\partial^{2} u}{\partial x^{2}} \frac{\partial^{2} v}{\partial x^{2}}+\frac{\partial^{2} u}{\partial y^{2}} \frac{\partial^{2} v}{\partial y^{2}}+2 \frac{a^{2} u}{\partial x y y}\right]\right\} d x$

Now on, we denote by $H$ the Hilbert $H^{2}(1) \cap H_{0}^{1}(2)$ provided with the norm $\|\cdot\| H^{2}(s)$. Then, we might define the weak formulation of (1) as follows : FIND $u \in H^{2}(\Omega)$ with $y_{0} u:=\operatorname{tr} u=g$, on $y$, such that.

$$
B(u, v)=\int_{\Omega} f v d x+\int_{\partial \Omega} h_{1} \frac{\partial v}{\partial n} d T
$$

for all $\vee \in H$. Here, $y_{0}:=t r$ stands for the trace operator of order 0 $[5]$.

Obviously, we can not apply lemma 1 to the above formulation. Nevertheless, since $g \in H^{3 / 2}\left(\partial_{3}\right)$, the trace theorem implies the ex istenco of a function $\tilde{w} \in H^{2}(n)$ such that $\gamma \tilde{w}:=\operatorname{tr} \tilde{w}=g$, on $\alpha_{n}$. So, if we set $\ddot{u}=u-\tilde{w}$, then it is clear that $\tilde{u}^{0} \in H$. Consequntly, we consider instead of (8) the following equivalent formulation: FIND ij $\in H$ such that

for all $\vee \in H$.

$$
B(u \tilde{u}, v)=\int_{\Omega} f v d x+\int_{\partial \Omega} h_{1} \frac{\partial v}{\partial n} d \tau-B(w, v)
$$

Our aim here is to prove the $H$ - ellipticity of $B$ in a simpler way than that usually given in the literature (sce, e.g., [6]: Pag. 395). For this, we need the following result. 
LEIIIT 2

Let. $|\cdot|_{H}(y)$ be the usual seminorm in $H^{*}(u)$, i.e.

$$
|v|_{H^{2}(\Omega)}^{2}-|\alpha|_{=2}^{i}\left\|0^{x} v\right\|_{L^{2}(a)}^{2}
$$

far all $\vee \in H^{2}(3)$. Ihen, there exists a positive romstant $f_{\text {an }}$ that

$$
|v|_{H^{2}(n)}^{?} \geq C\|v\|_{H^{2}(\Omega)}^{?}
$$

iar all V F H.

Pritrif:

liven $v \in H:=H^{2}(\Omega) \cap H_{n}^{1}(\zeta)$, we define $f_{V}-A v$ and consider the lallowing Dirichlet problem with homogeneous data: FIND LE H that

$$
\int v u \cdot v w d x-\iint_{\Omega} f^{w d x}
$$

ini $\rightarrow 11$ WEH

since I G. $L^{2}(\Omega)$, it is very well kncwn that (1) has a unique oniution u which arourding to (5) (lemma 1) satisfine

$$
\|1,\|_{H}(0)<\frac{1}{\beta}\left\|f_{v}\right\|_{L} ?^{2}(n)
$$

for some positive constant $\beta$. But, since v E H2(s), the cirrem's socard inemtity provides that, in fact, the unique solution of (1l) is vitself. linrefore, instead of (]2) we can write

$$
\|v\|\left\|^{1}(\Omega) \times \frac{1}{\beta}\right\| \wedge v \|_{L^{2}(\Omega)}
$$

Homer, we deduce that

$$
\|v\|_{H^{2}(\Omega)}^{2} \leqslant \frac{1}{B^{2}}\|\Delta v\|_{L^{2}(\Omega)}^{2}+\|\left. v\right|_{H^{2}(0)} ^{2}
$$

and since $\|A v\|_{L^{2}(\Omega)}^{2} \leqslant 2 \|\left. v\right|_{H^{2}(\Omega)} ^{2}$, we conelude that

$$
C\|v\|_{H^{2}(0)}^{2} \leqslant \|\left. v\right|_{H^{2}(\Omega)} ^{2}
$$

lar rll v G.H, with C. $\left(1+\frac{2}{\beta^{x}}\right)^{-1}$.

Fimaly, the $H$ - etliptictty of $B$ is a dircel compeguence of the rhouve lortuna. In fact, from (7) and (10) we ontaim 


$$
\begin{aligned}
B(v, v)= & \sigma|| w \|_{L^{2}(\Omega)}^{2}+(1-\sigma)|v|_{H^{2}(\Omega)}^{2} \\
& \geqslant(1-\sigma)|v|_{H^{2}(\Omega)}^{2} \\
& \geqslant c(1-\sigma)\|v\|_{H^{2}(\Omega)}^{2}
\end{aligned}
$$

for all $\vee \in H$.

Now, we turn our attention to the Neunam problem (?) which also arises in the theory of thin plates [3]. It follows easily from (2) and (6) that the weak formulation of (2) is given by: $F\left[N \mid\right.$ II $F H^{2}(\Omega)$ such that

$$
B(u, v)=F(v)
$$

for all $\vee \in H^{2}(\Omega)$, where $F$ is the bounded linear fumctimal given by

$$
F(v):=\int_{\Omega} f v d x+\int_{\partial n} v h_{2} d T+\int_{\lambda} \frac{\partial v}{\partial n} h_{1} d l
$$

On the other hand, as indicated in $|6|$, it is clearly seen that any $i$ inear conbination of the polynomials $1, x$ and $y$ is a solution of the momogeneous case for (13). So, in order to get unigunness certain normalizatiun conditions are required.

We define the functionals

$$
\begin{aligned}
& r_{1}, r_{2}, f_{3}: H^{2}(\Omega) \rightarrow R \\
& f_{1}(v):-\int_{\Omega} v d x, f_{2}(v):=\int_{\Omega} x v d x \\
& f_{3}(v):=\int_{\Omega} y v d x
\end{aligned}
$$

simce $\Omega$ is bounded, it is clear that $f_{j}$ is a bundrel linear furctional for all i- $1,2,3$.

Thus, we consider instead of (2) the following BVP

$$
\begin{aligned}
& b^{2} u=r, \text { in } n \\
& M[u]=n_{1}, N[u]=n_{2}, \text { on } a x^{\prime} \\
& f_{i}(u)=A_{i}, i=1,2,3
\end{aligned}
$$

where $A_{1}, A_{2}, A_{3}$ are given real numbers. This means thilt (13) is replacod by:

FINI] $u \in H^{2}(\Omega)$ with $f_{i}(u)=A_{j}$, $i-1,2,3$ such that

$$
B(u, v)=F(v)
$$


for al 1 v $\in H^{2}(\Omega)$

Now, for a given $\vee \in H^{*}(\Omega)$, there exists a unique nolymomial $P(x, y):=a+b x+c y$ such that

$$
f_{i}(v)=f_{i}(p)+i=1,2,3
$$

In fart, if we write out the equation (16), then we nhtain the far amm system whose determinant is always different from zero.

So, if $H_{E}^{2}(s)$ denotes the space

$$
H_{F}^{2}(\Omega):-\left\{w \in H^{2}(\Omega): f_{i}(w)-0, i-1,2,3\right\}
$$

then. it is obvious that $\tilde{v}:=v-p$ belongs to $H_{E}^{2}(\Omega)$ for all $v \in H^{2}(\Omega)$. Therefore, by replacing $v=\tilde{v}+p$ in $(15)$, noting that $B(p, v)=B(v, p)=0$ for all $p, v$, and assuming that $f, h_{1}, h_{2}$ satisfy the compatibility condisions

$$
F(v)-0 \text {, for } v=1, x, y
$$

We obtain the following weak formulation, equivalent to (15):

FINI] if $\mathrm{H}_{\mathrm{E}}{ }^{2}(\Omega)$ such that

$$
B(i \mathrm{i}, v)=F(v)
$$

fur nll $\vee \in H_{E}^{2}(\Omega)$.

It is inportant to note that if $\vec{p}$ is the unique polymomial satisfying $f_{i}(\tilde{p})=A_{j}, i=1,2,3$, then $u:=\tilde{u}+\tilde{p}$ is the corresponding solution of (15).

Our next aim is to show that the $H_{E}^{2}(\Omega)$ - fllipticity of the bilinear form Bes a simple corollary of the following generalized Poincare inciual ity $\lceil 5\rceil$.

ihearem I

Denote by $\Gamma_{k-1}$ the set of all polymutials in $k^{2}$ of degreo $<k-1$. lot $T$ be a finite ramily of bounded tinear functimals on the sobolev space $H^{k}, "(s)$, with the property

$$
v \text { E. } P_{k-1} \text { and } f(v)=0 \text {, for all } f \text { f } \Gamma \text { implies } v=0
$$

Suppose $\Omega$ is a $C^{0,1}$ domain. Then

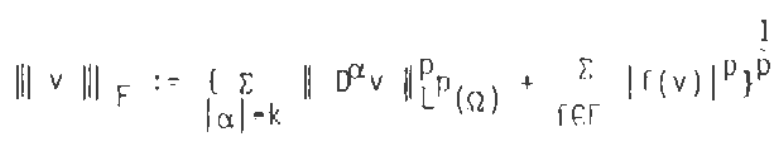


is a norm in $H^{k, p}(\Omega)$ which is equivalent to the usual norro $\|v\|_{H^{k}}, p_{(\Omega)}$. i.p. there exist positive constants $C_{1}, C_{2}$ so that

$$
C_{1}\|v\|_{H^{k}, p_{(\Omega)}} \leqslant\|v\|_{F} \leqslant C_{2}\|v\|_{H} k, p(\Omega)
$$

for tili $\vee \in H^{k}, P(\Omega)$.

Pronf:

$$
\text { See }|5| \text { : Theorem } 5.4 .2 \text {. }
$$

In the particular case of $k=p=$ ?, we already noted that rur. fanily $\left.F:=\mathrm{ff}_{1}, \mathrm{f}_{2}, \mathrm{f}_{3}\right\}$ satisfies clearly the property (18). Therafur.. there $\mathrm{exists} \mathrm{C}_{1}>0$ such that

$$
\|v\|_{F} \geqslant C_{1}\|v\|_{H}(\Omega)
$$

for all ve $H^{2}(\Omega)$, where

$$
\|v i\|_{f}^{2}:=|v|_{H^{2}(\Omega)}^{2}+\sum_{i=1}^{3}\left|f_{i}(v)\right|^{2}
$$

It fol lows from (2l) that $\left\|\left.\left|v \|_{F}=\right| v\right|_{H^{2}(\Omega)}\right.$, for all $v \in H_{E}^{2}(\Omega)$. Hence. we conclude that

$$
\begin{aligned}
B(v, v) & =0\|N\|_{L}^{2}(\Omega)+(1-0)|v|_{H^{2}(0)}^{2} \\
& \geqslant(1-0)\|v\| \|_{F}^{2} \\
& \geqslant c_{1}(1-0)\|v\|_{H^{2}(\Omega)}^{2}
\end{aligned}
$$

ior ali $\vee \in H_{!}^{2}(0)$. 


\section{REFERENCES}

[1] AZIZ, A.; BABUSKA, 1.: "Survey lectures on the mathematical foundations of the finite element method". In the mathematical foundations of the finite element method with applications to partial dif ferential equations, A. Az is ed., Academic Press, New York, 1972.

[2] CIARLET, P.: "The finite element method for elliptic problems". North-Holland Publishing Company, 1978.

[3] FICHERA, G. : "Linear elliptic differential systems and eigenvalue problems". Lecture notes in mathematics 8, Springer-Verlag, Berlin, 1965.

[4] FRIEDMAN, A. : "Partial differential equations". Robert E. Krieger Publishing Company, Inc., 1969.

[5] KUFNER, A.; JOHN, 0.; FUCIK, S. : "Function spaces". Prague, Academia, 1977.

[6] REKTORYS, K. : "Variational methods in mathematics, science and engineering". D. Reidel Publishing Co., Dordrecht, Holland, 1980.

Gabriel N. Catica

Departamento de Matemática

Universidad de Concepción

Casilla 2017

Concepción - CHILE 